\title{
Sequential and non-sequential simulation of volume holographic gratings
}

\author{
Moritz Kick ${ }^{1,2^{*}}$ (D) Reinhold Fieß ${ }^{1}$ and Wilhelm Stork ${ }^{2}$
}

\begin{abstract}
Background: In the development process of holographic displays like holographic Head-Mounted Displays (hHMD) the simulation of the complete optical system is strongly required. This especially includes the correct behaviour of the volume holographic grating (VHG) in terms of its optical function and its diffraction efficiency. The latter is not supported by the current version of Zemax ${ }^{\circledR}$ OpticStudio 17, one of the most popular optic simulation tools.

Methods: To solve this problem we implemented a $C_{++}$code for each raytracing mode of Zemax ${ }^{\oplus}$, namely the sequential and non-sequential. The $C_{+}+$code calculates the grating vector for every single ray traced. Based on the $\mathrm{k}$-sphere formalism the propagation direction of the diffracted light is determined. Furthermore, its diffraction efficiency is defined according to Kogelnik's coupled-wave theory. The $C_{++}$code is compiled and linked into Zemax ${ }^{\otimes}$ using the Windows Dynamic Link Library (DLL).

Results and discussion: The aforementioned DLL enables the simulation of planar and arbitrarily spherical curved VHG and their diffraction efficiency within Zemax ${ }^{\circledR}$ OpticStudio. This allows the fast, easy and reliable simulation of optical systems which include holograms or holographic optical elements, e.g. hHMD. Especially the simulation of VHG in non-sequential mode can be helpful in order to identify possible stray light paths.
\end{abstract}

Conclusion: The implemented $\mathrm{C}++$ code enables the user to simulate VHG and its diffraction efficiency within Zemax ${ }^{\oplus}$ Optic Studio.

Keywords: Holography, Holographic optical elements, Hoe, Head mounted display, Diffraction efficiency, Kogelnik, Zemax

\section{Background}

Recently, new display technologies using holographic approaches have been reported [1-4]. VHG have several advantages compared to conventional optics: they can be space-saving, lightweight and they offer a high degree of freedom in the optical design process. On the other hand their strong dependency on wavelength and incidence angle can be problematic if a robust and reliable optical design is required. Additionally, holographic optical systems tend to suffer from stray light due to reflections at glass-air boundaries and multiple interactions with the VHG. In order to avoid disturbing stray light paths it is crucial to simulate VHG and their diffraction efficiencies during the design process. Unfortunately,

${ }^{*}$ Correspondence: moritz.kick@de.bosch.com

'Robert Bosch GmbH, Robert-Bosch-Campus, 71272 Renningen, Germany

${ }^{2}$ Karlsruher Institut für Technologie, Institut für Technik der

Informationsverarbeitung, Engesstraße 5, 76131 Karlsruhe, Germany most of the commercially available optic simulation tools like Zemax ${ }^{\circ}$ OpticStudio don't provide this possibility. There have been different approaches to overcome this problem. In Ref [5-7] the Born approximation is used to estimate the diffraction efficiency. This approximation assumes a weak interaction of the lightfield with the VHG. As in display technologies high diffraction efficiencies are favourable, simulations based on the Born approximation lead to inaccurate results. A suitable description of highly efficient (planar) VHG is given by Kogelnik's coupledwave theory [8]. In Ref [9] aperiodic and multiplexed VHG are analyzed based on the coupled-wave theory within Zemax. In order to simulate non-planar gratings the hologram plane is split into a large number of sampling zones. For every sampling zone the assumption of a planar grating holds [10] if the grating vector is slowly varying. Finally, the diffraction efficiency is averaged over all sampling zones. 
This paper describes a simple method of simulating arbitrarily spherical curved and planar VHG. For every ray traced the grating vector is calculated locally and the diffraction efficiency is calculated based on coupled-wave equations. The new method is integrated into the commercially available optic simulation tool Zemax ${ }^{\oplus}$ using a DLL for each, the sequential and non-sequantial raytracing mode. Thereby, it enables the use of a manifold of optimization tools included in Zemax ${ }^{\odot}$ during the design process of optical systems like hHMD. In Fig. 1 an exemplary hHMD system is shown. A laser unit is used as the light source and a deflector (e.g. mirror) guides the light to an holographic optical element (HOE), which is described in greater detail in the following section. In this example the HOE is used to diffract the light into the direction of the observer. As the HOE is transparent, the observer is able to see the surroundings and the information provided by the hHMD simultaneously.

\section{Methods}

\section{Holographic optical elements}

HOE are (V)HG utilized as optical elements like lenses or mirrors. They are space-saving, lightweight, wavelengthand angle-selective, transparent and potentially low-cost. Additionally HOE do have the advantage of adding freedom to the optical design process, as incidence and exit angle can be chosen independently. In the recent past new holographic materials have been introduced to the market [11-15]. They allow the recording of color VHG without the need of chemical or thermal processing, which makes them suitable for mass-production. Therefore, HOE could be the key to satisfy the growing demand for compact and lightweight HMD systems.

HOE can work in transmission or reflection, as depicted in Fig. $2 a$ and $b$. The reference wave used to reconstruct the HOE (red, dashed) is defined by its wave vektor $\mathbf{k}_{R}$ and hits the HOE from the left hand side. Inside the HOE the reference wave interacts with the plane VHG, which itself is defined by the grating vector $\mathbf{k}_{\mathrm{G}}$. Some part

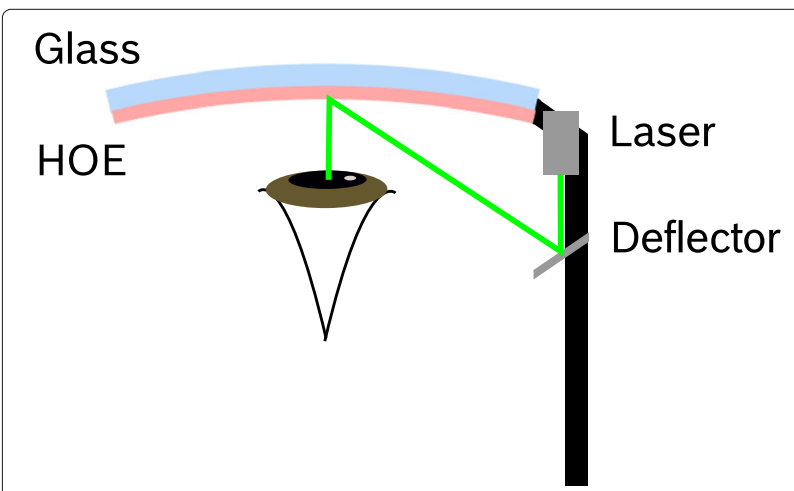

Fig. 1 Example for a HMD system with laser source, beam deflector and $\mathrm{HOE}$. The HOE is used to diffract the light to the observer of the reference wave is diffracted into the object wave $\left(\mathbf{k}_{\mathrm{O}}\right.$, green, solid). The not-diffracted part propagates undisturbed (blue, dotted). When $\mathbf{k}_{\mathrm{R}}$ and $\mathbf{k}_{\mathrm{G}}$ are known, $\mathbf{k}_{\mathrm{O}}$ can be calculated using the $\mathrm{k}$-sphere formalism [6]

$$
\begin{aligned}
\mathbf{k}_{O} \cdot(\hat{\mathbf{x}}+\hat{\mathbf{y}}) & =\left(\mathbf{k}_{R}+\mathbf{k}_{G}\right) \cdot(\hat{\mathbf{x}}+\hat{\mathbf{y}}), \\
\left|\mathbf{k}_{O}\right| & =\frac{2 \pi}{\lambda},
\end{aligned}
$$

with $\hat{\mathbf{x}}$ and $\hat{\mathbf{y}}$ denoting the unit base vectors in the hologram plane. Based on these equations the propagation direction of the diffracted light rays is calculated within the DLL.

\section{Kogelnik's coupled-wave theory}

Besides the propagation direction the diffraction efficiency has to be calculated. The diffraction efficiency $\eta$ is defined as the fraction $I_{1}$ of the incident reference wave $I_{0}$ which is diffracted into the object wave

$$
\eta=\frac{I_{1}}{I_{0}}
$$

For plane VHG the rigorous coupled-wave analysis can accurately predict the diffraction efficiency. Unfortunately, solving these equations can be very time consuming. Instead, Kogelnik's coupled-wave theory is an approximate solution of the coupled-wave theory and is frequently used to predict the diffraction efficiencies of VHG. The approximate solutions accurately predict the diffraction efficiency if the following requirements are fulfilled [8]

- hologram thickness $d \gg \lambda$, the used wavelength,

- the light field is monochromatic,

- reconstruction of the hologram near the Bragg angle,

- only reference and object wave are present,

- the holographic grating is sinusoidal,

- the light field is polarized perpendicular to the plane of incidence.

These requirements are fulfilled for the use of VHG as HOE in display technologies like hHMD. In the case of planar reference and object recording waves, the grating vector is constant within the entire hologram volume and is defined by

$$
\mathbf{k}_{G}=\mathbf{k}_{O, r e c}-\mathbf{k}_{R, r e c},
$$

which is also visualized in the subset of Fig. 3. If one or both waves do have a spherical shape, the grating vector will vary over the hologram volume: $\mathbf{k}_{\mathrm{G}}=\mathbf{k}_{\mathrm{G}}(x, y, z)$. But if only a small enough area is investigated, even for strong curvatures the assumption of a planar grating holds and Eq. 3 can be used to locally calculate the grating vector. With the assumptions made above, Kogelnik was able to find analytical solutions for the the coupled-wave equations $[8,9,16]$. As different boundary conditions 

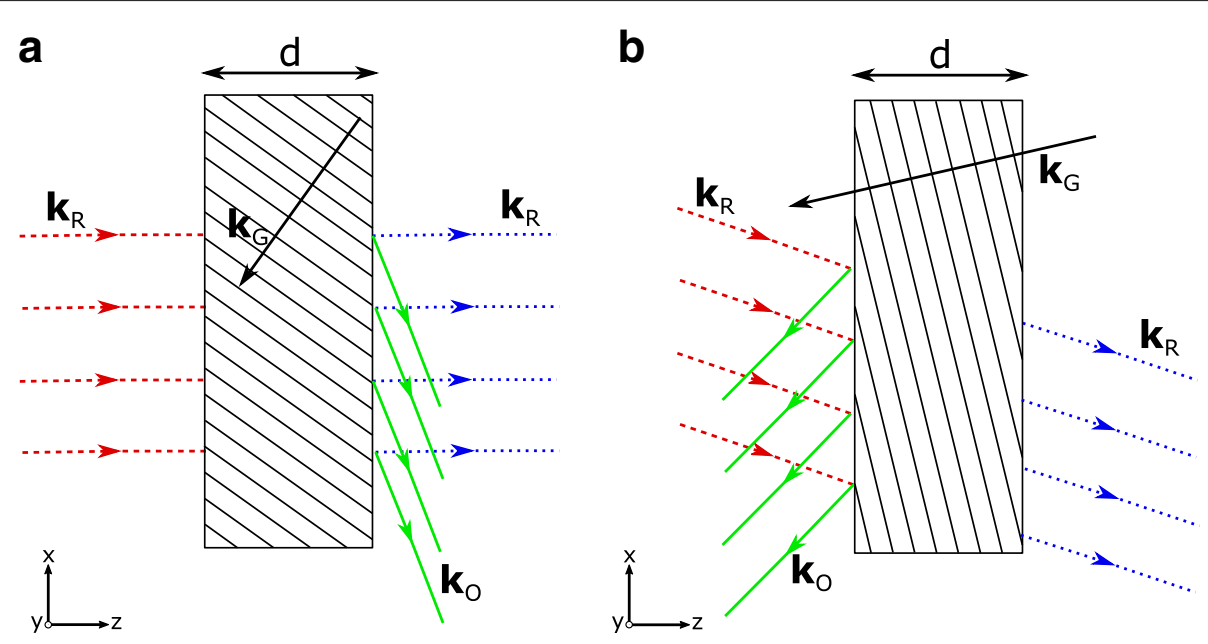

Fig. 2 Example for transmission and reflection HOE a HOE that works in transmission. $\mathbf{b} \mathrm{HOE}$ that works in reflection. $\mathbf{k}_{R}, \mathbf{k}_{\mathrm{O}}$ and $\mathbf{k}_{\mathrm{G}}$ denote the wave vector of the reference and object beam and the grating vector, respectively. $d$ is the thickness of the HOE. The light hits the HOE from the left hand side (red, dashed), interacting with the VHG. Thereby, some part of the reference wave is diffracted into the first order, which is called the object wave (green, solid). The non-diffracted part propagates in the same direction as $\mathbf{k}_{\mathrm{R}}$ (blue, dotted)

are used for transmission and reflection holograms, these cases have to be treated seperately. However, to allow for a clear representation of the diffraction efficiency in both cases, the following auxiliary parameters are defined $[8,16]$

$$
\begin{array}{lll}
\boldsymbol{\sigma}=\mathbf{k}_{R}+\mathbf{k}_{G}, & c_{O}=\frac{\boldsymbol{\sigma} \cdot \mathbf{n}}{\left|\mathbf{k}_{R}\right|}, & c_{R}=\frac{\mathbf{k}_{R} \cdot \mathbf{n}}{\left|\mathbf{k}_{R}\right|}, \\
\vartheta=\frac{\mathbf{k}_{\mathrm{R}}^{2}-\boldsymbol{\sigma}^{2}}{2\left|\mathbf{k}_{R}\right|}, & \xi=\frac{\vartheta \cdot d}{2\left|c_{O}\right|}, & v=\frac{\pi \cdot \Delta n \cdot d}{\lambda \cdot \sqrt{c_{O} \cdot c_{R}}},
\end{array}
$$

where $\mathbf{n}$ is the surface normal of the hologram plane and $\Delta n$ is the refractive index modulation of the holographic material.

\section{Transmission holograms}

According to Kogelnik the diffraction efficiency $\eta_{T}$ of a lossless transmission-type volume HOE is given by [8]

$$
\eta_{T}=\frac{\sin ^{2} \sqrt{v^{2}+\xi^{2}}}{1+\frac{\xi^{2}}{v^{2}}} .
$$

A diffraction efficiency of 1 is possible if $\xi=0$ holds, which implies that the detuning parameter $\vartheta=0$. This is the case if the VHG is reconstructed under perfect Bragg-condition [17]:

$$
2 \Lambda \sin \Theta=m \cdot \lambda
$$

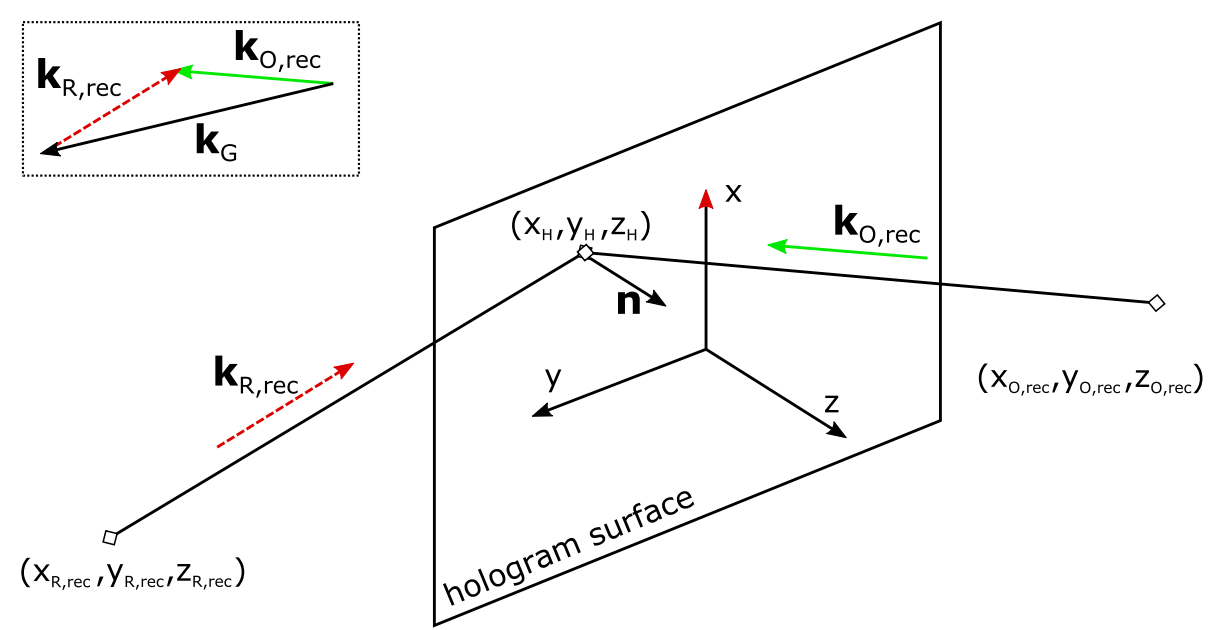

Fig. 3 Definition of optical function. View of two point sources which define the optical function of the HOE and the interception point with the hologram surface for a single ray. In the subset in the left upper corner Eq. 3 is visualized 
with the reconstruction ray impinging on the VHG with an angle $\Theta$, the grating spacing $\Lambda=\frac{2 \pi}{\left|K_{G}\right|}$ and integer value $\mathrm{m}$, specifying the diffraction order. In Kogelnik's coupled wave theory higher diffraction orders are neglected, therefore m equals 1 .

Equation 4 will be used to calculate the diffraction efficiency of transmission-type $\mathrm{HOE}$ in the $\mathrm{C}++$-code for sequential and non-sequential mode.

\section{Reflection holograms}

For lossless reflection-type volume HOEs the diffraction efficiency $\eta_{R}$ is given by [8]

$$
\eta_{R}=\left(1+\frac{1-\frac{\xi^{2}}{v^{2}}}{\sinh ^{2} \sqrt{v^{2}-\xi^{2}}}\right)^{-1} .
$$

Here, special attention has to be paid as the argument of the square root can be negative. In these cases, the hyperbolic sine has a complex argument and therefore will transfer into a sine-function with a real-valued argument. Similarly to transmission $\mathrm{HOE}$, reflection HOE are most efficient if $\vartheta=\xi=0$ holds. Equation 6 will be used to calculate the diffraction efficiency of reflection-type $\mathrm{HOE}$ in the $\mathrm{C}++$-code for sequential and non-sequential mode.

\section{Implementation of the DLL}

The goal of this work is to enhance the commercially available optic simulation tool Zemax ${ }^{\circ}$ OpticStudio to allow the fast and easy simulation of optical systems which include arbitrarily spherical curved VHG. Therefore, for the sequential and non-sequential raytracing mode a $\mathrm{C}++$-code has been implemented which is compiled to a DLL and linked into Zemax ${ }^{\circ}$.

When integrating a DLL into Zemax ${ }^{\circ}$ this gives access to a manifold of parameters as described in Ref [18]. The following will be used in this work

- unit vector $\hat{\mathbf{k}}_{\mathrm{R}}$ of the incoming ray,

- wavelength $\lambda$ of incoming ray (note that $\mathbf{k}_{\mathrm{R}}=\frac{2 \pi}{\lambda} \cdot \hat{\mathbf{k}}_{\mathrm{R}}$ ),

- interception point $\left(x_{H}, y_{H}, z_{H}\right)$ of the incoming ray with the hologram surface (see Fig. 3),

- refractive index of the materials surrounding the hologram surface.

Additionally, user-defined paramters specify the optical function and the material properties of the hologram. The coordinates $\left(x_{R, \text { rec }}, y_{R, \text { rec }}, z_{R, \text { rec }}\right)$ and $\left(x_{O, \text { rec }}, y_{O, \text { rec }}, z_{O, \text { rec }}\right)$ define the point sources of spherical reference and object wave used during recording. Note that if a plane wave is preferred, the distance of the point source should be located in great distance to the hologram surface (e.g. $\sqrt{x_{R, \text { rec }}^{2}+y_{R, \text { rec }}^{2}+z_{R, \text { rec }}^{2}} \gg 10^{6} \mathrm{~mm}$ for a plane reference wave). Furthermore, the wavelength $\lambda_{\text {rec }}$ used during recording, the thickness $d$ of the hologram as well as the average refractive index $n$ and its modulation $\Delta n$ need to be specified.

When all parameters are set the simulation can be started. Everytime the DLL is called the following steps are performed

- Based on the geometry depicted in Fig. $3 \mathbf{k}_{R, \text { rec }}$ and $\mathbf{k}_{O, \text { rec }}$ are calculated as follows

$\mathbf{k}_{\mathrm{i}, \mathrm{rec}} \cdot \hat{\mathbf{x}}=\left(x_{H}-x_{i, \text { rec }}\right) / N_{i}$,

$\mathbf{k}_{\mathrm{i}, \text { rec }} \cdot \hat{\mathbf{y}}=\left(y_{H}-y_{i, \text { rec }}\right) / N_{i}$,

$\mathbf{k}_{\mathrm{i}, \text { rec }} \cdot \hat{\mathbf{z}}=\left(z_{H}-z_{i, \text { rec }}\right) / N_{i}$,

$N_{i}=\frac{\lambda_{\text {rec }}}{2 \pi} \sqrt{\left(x_{H}-x_{i, r e c}\right)^{2}+\left(y_{H}-y_{i, r e c}\right)^{2}+\left(z_{H}-z_{i, r e c}\right)^{2}}$,

with $i=R, O$.

- $\mathbf{k}_{\mathrm{R}}, \mathbf{k}_{R, \text { rec }}$ and $\mathbf{k}_{O \text {,rec }}$ are refracted according to Snell's law,

- $\mathbf{k}_{\mathrm{G}}$ is calculated based on Eq. 3,

- $\mathbf{k}_{\mathrm{O}}$ is calculated based on Eq. 1 ,

- depending on the position of the given point sources a distinction of cases between transmission and reflection holograms is made,

- the diffraction efficiency is calculated based on Eq. 4 in case of a transmission and Eq. 6 in case of a reflection hologram,

- $\mathbf{k}_{\mathrm{O}}$ is refracted according to Snell's law,

- the diffraction efficiency and the unit vector $\hat{\mathbf{k}}_{\mathrm{O}}$ are transferred to Zemax.

The data transfer between the DLL and Zemax differs for sequential and non-sequential mode, as different data structures are predefined by Zemax ${ }^{\oplus}$. The parameters $U D \rightarrow$ rel_surf_tran and $U D \rightarrow l, m, n$ serve to transfer the diffraction efficiency and the propagation direction of the diffracted light in sequential mode, respecively. In the non-sequential mode, we made use of the parameters data[30], data[32], data[33] and data [34]. Note that the whole procedure is repeated for every single ray that interacts with the hologram surface. In Zemax ${ }^{\circ}$ rays interact with a surface at a single (intercept) point. To model the behaviour of a volume hologram, a single incident ray's interaction with the VHG is assumed to be limited to a small suface region of the VHG, in which the grating can be assumed to be plane. However, the orientation of the grating vector can vary across the hologram surface. As previously mentioned this enables us to simulate not only plane, but also any spherical curved VHG.

\section{Results and discussion}

In this section examples of use of the DLL in the sequential and non-sequential mode of Zemax are given. They prove the benefits of the developed DLL when designing holographic optical systems in sequential mode and 

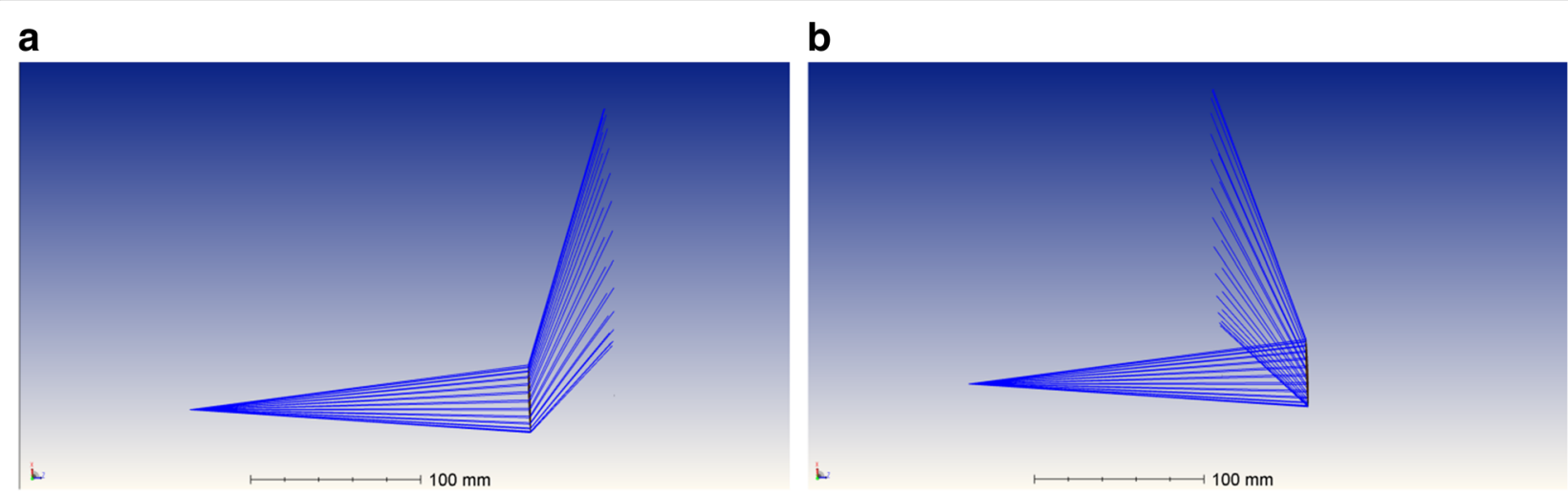

Fig. 4 Shaded Model of a transmission-type a and reflection-type HOE $\mathbf{b}$ simulated in Zemax ${ }^{\circledast}$. The parameters that define the HOE are given in Table 1

evaluating their behaviour in terms of parasitic effects in non-sequential mode.

\section{Sequential raytracing mode}

The sequential mode is used to design and optimize optical systems, for example hHMD systems. By the use of the DLL the design process is greatly accelerated, as the characteristica of the HOE can be considered instantly.

The DLL is integrated into Zemax ${ }^{\ominus}$ as a User Defined Surface (UDS). Once loaded the user-defined parameters have to be set.

In Fig. 4 two examples of Zemax ${ }^{\circledR}$ Shaded Models are depicted: a transmission a) and reflection b) HOE which are defined by the parameters given in Table 1 . For example, the reflecion-type HOE could be used in a hHMD system as depicted in Fig. 1. The Zemax operand $I M A E$ gives access to the transmission of a surface. Therefore, it allows to read out the overall diffraction efficiency of the hologram. Various analysis and optimization tools integrated into Zemax $^{\oplus}$ enable the fast and easy investigation of the HOE. Exemplary a selction of Zemax ${ }^{\odot}$ Universal Plots is depicted in Fig. 5. In Fig. 5a the diffraction efficiency $\eta_{T}$ of the transmission-type $\mathrm{HOE}$ is plotted against its thickness $d$ when reconstructed under perfect Bragg-condition. The expected sinusoidal behaviour is observed, with maximum efficiency $\eta_{T}=1$ for $d \approx 5 \mu \mathrm{m}, 15 \mu \mathrm{m}, 25 \mu \mathrm{m}, \ldots$ (compare Eq. 4). Figure 5b shows that the diffraction efficiency decreases if the reconstruction wavelength $\lambda$ differs from the recording wavelength $\left(\lambda_{\text {rec }}=0.55 \mu \mathrm{m}\right)$, when all other parameters are fixed. In Fig. $5 c$ and $d$ the same plots are depicted for the reflection hologram. In accordance with the Bragg-reflection the diffraction efficiency of a reflection holograms increases with its the thickness $d$ (see Fig. 5c). Finally, when comparing Fig. 5b and $d$ a much steeper slope can be found for reflection holograms. In fact, that's the reason why reflection holograms are named to be wavelength-selective. All simulation results are as expected and prove that the DLL enables the reliable simulation of VHG.

The examples should give the reader an idea how the DLL can be used to optimize HOEs in sequential mode. Integration of the HOE into a more complex optical system is very straightforward. By the use of the user-defined parameters and the operand IMAE merit-functions can be defined. This enables the user to simultaneously optimize the optical system and the HOE.

\section{Non-sequential raytracing mode}

In the non-sequential mode of Zemax ${ }^{\circledR}$ rays don't hit the optical surfaces in a predefined order, but can intersect the same surface many times and multiple surfaces in any order. Furthermore, an intersecting ray can split up into many rays according to the laws of refraction, reflection and diffraction. This enables us to consider the not-diffracted part, by adding an additional order with efficiency $\eta_{n d}=1-\eta_{i}, i=T, R$. The non-sequential

Table 1 User-defined hologram parameters

\begin{tabular}{lll}
\hline & Transmission & Reflection \\
\hline$x_{R, r e c}[\mathrm{~mm}]$ & -40 & -40 \\
$y_{R, r e c}[\mathrm{~mm}]$ & 0 & 0 \\
$z_{R, \text { rec }}[\mathrm{mm}]$ & -20 & 20 \\
$x_{0, \text { rec }}[\mathrm{mm}]$ & 0 & 0 \\
$y_{O, \text { rec }}[\mathrm{mm}]$ & 0 & 0 \\
$z_{0, \text { rec }}[\mathrm{mm}]$ & -200 & -200 \\
$\lambda_{\text {rec }}[\mu \mathrm{m}]$ & 0.550 & 0.550 \\
$d[\mu \mathrm{m}]$ & 5 & 9 \\
$n$ & 1.5 & 1.5 \\
$\Delta n$ & 0.05 & 0.05 \\
\hline
\end{tabular}

The parameters which define the optical function of the holograms used in the given examples 


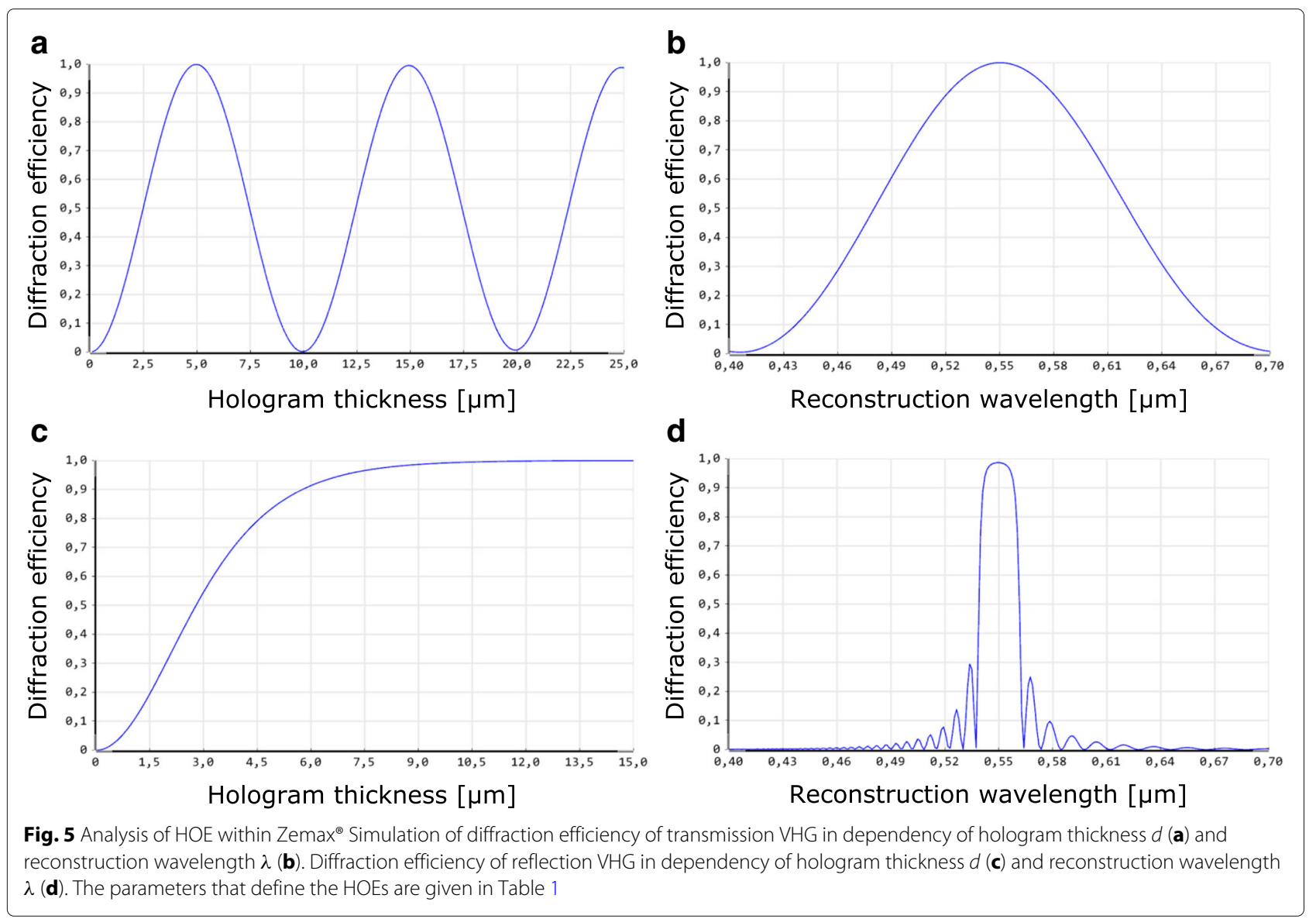

mode can be used to identify possible parasitic effects of the optical system previously developed in the sequential mode. That means we focus on effects like double images and stray light.

To include the DLL into the non-sequential mode it is used as a diffractive property of an existing surface, e.g. the Hologram Lens. As an example, a reflection hologram defined by the same parameters as in the sequential mode is used (see Table 1).

A NSC Shaded Model is shown in Fig. 6a. In addition to the diffracted light the reflected and transmitted part is visible. Furthermore, a possible stray light path is identified in transmission. It occurs due to a reflection at the front air-hologram boundary after the interaction with the HOE. To display rays with less intensity we reduced the Minimum Relative Ray Intensity from 8,0000E-03 to $8,0000 \mathrm{E}-07$. Subsequently, a lot of additional ray paths become visible (see Fig. 6b). Without the non-sequential investigation of an optical system it is nearly impossible to think of all possible stray light paths, even for a simple example like the one presented. With the Detector Rectangle, also shown in Fig. $6 \mathrm{~b}$ as an orange line, the a

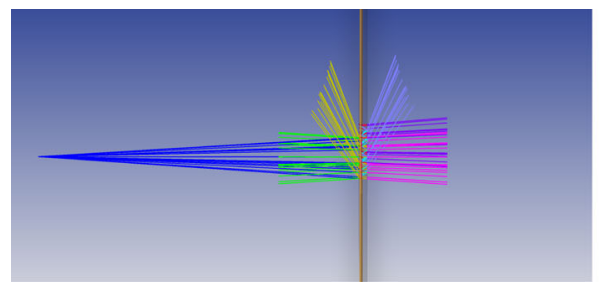

b

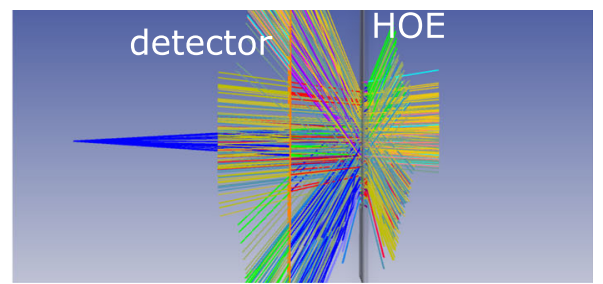

Fig. 6 NSC Shaded Model of the reflection hologram in Zemax ${ }^{\circledR}$ non-sequential mode. a The Minimum Relative Ray Intensity is set to 8,0000E-03. b The Minimum Relative Ray Intensity is set to 8,0000E-07 


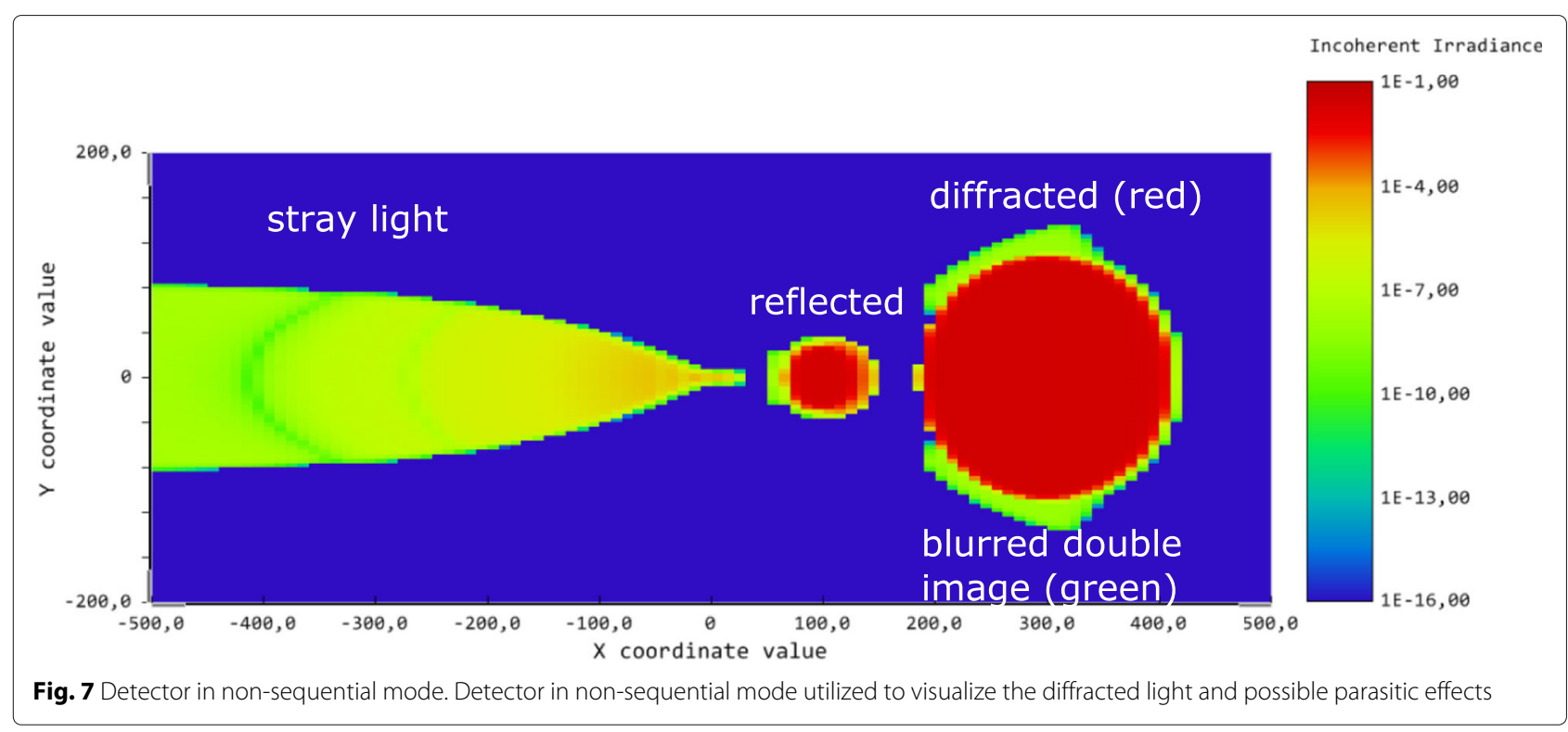

reflected light is displayed. For a hHMD system this could be the light which is seen by the observer. For the discussed setup the results are shown in Fig. 7. The big red spot on the right hand side is the diffracted light, which is the useful light. It is surrounded by a blurred double image (green). This double image is generated due to multiple reflections of the diffracted light at the hologramair boundaries. The central spot is the (fresnel) reflected light. On the left hand side a large tail of stray light is observed. It occurs due to light that is reflected from the rear hologram-air boundary and afterwards diffracted by the hologram in reverse geometry. In case of a hHMD systems the stray light, the double image and even the fresnel reflected part could be a potential problem. By changing the parameters or the geometry of the hologram, by using anti reflection coatings or adding apertures into the optical setup, these effects could be suppressed.

It has been shown that the implemented DLL enables the simulation of parasitic effects like double images and stray light in the non-sequential mode. This is a crucial task when evaluating holographic optical system like hHMD.

\section{Conclusions}

This paper dealt with an extension written for the optical simulation tool Zemax ${ }^{\odot}$ OpticStudio. For both raytracing modes, namely the sequential and the non-sequential mode, a DLL has been implemented which mimics the behaviour of an HOE including its diffraction efficiency. The basic concept of the underlying coupled-wave theory has been introduced. It was shown how to integrate the DLL into Zemax ${ }^{\circledR}$ and examples of use for both raytracing modes were given. Especially the possibility of simulating HOEs with proper diffraction efficiencies in the non-sequential mode, which enables the simulation of stray light effects or double images, can be of great use for future work. It also should be mentioned that combining the DLL with the useful optimization tools of Zemax ${ }^{\oplus}$ simplifies the design process of holographic optical systems. The presented DLL is restricted to simulate HOEs defined by diverging waves or plane (TypeI-HOE). For the case of a converging reference or object wave (TypeII-HOE) an additional DLL has been implemented. It can be used exactly in the same manner as the described DLL with the only difference that one point source is now treated as a point sink (compare to TypeI- and TypeII-HOE of Zemax $^{\oplus}$, mentioned in ref. [18]).

It is left for the future to adapt the DLL to spherical or cylindrical surfaces as currently the surface of the HOE needs to be plane. Furthermore, defining the optical function of the HOE by the use of Zernike polynomials instead of two spherical waves could lead to more complex optical functions for the simulated HOE. Subsequently, aberrations of the optical system could be simulated and corrected by adjusting the HOE function.

\section{Abbreviations}

DLL: Dynamic link library; hHMD: Holographic head-mounted display; HOE: Holographic optical element; VHG: Volume holographic grating; UDS: User defined surface

\section{Acknowledgements}

This work was supported by Prof. Dr. Norbert Lindlein (Universität Erlangen) and Patrick Wissmann [6].

Availability of data and materials

The data has been provided in the manuscript.

\section{Authors' contributions}

MK implemented the $C_{++}$-code and wrote the manuscript. WS and RF made contribution to the development of the idea and read and approved the final manuscript. 


\section{Competing interests}

The authors declare that they have no competing interests.

\section{Publisher's Note}

Springer Nature remains neutral with regard to jurisdictional claims in published maps and institutional affiliations.

\section{Author details}

Received: 6 November 2017 Accepted: 25 April 2018

Published online: 16 May 2018

\section{References}

1. Kasai, I, Tanijiri, Y, Endo, T, Ueda, H: A practical see-through head mounted display using a holographic optical element. Opt. Rev. 8(4), 241-244 (2001)

2. Maimone, A, Georgiou, A, Kollin, JS: Holographic near-eye displays for virtual and augmented reality. ACM Trans. Graph. 36(4), 85-18516 (2017)

3. Hsieh, P-Y, Oi, R, Senoh, T, Sasaki, H, Ichihashi, Y, Okui, M, Huang, Y-P, Yamamoto, K, Wakunami, K: Projection-type see-through holographic three-dimensional display. Nat. Commun. 7, 12954-12961 (2016)

4. Hong, J, Yeom, J, Kim, Y, Park, J, Cho, J, Hong, S, Jung, K-M, Kang, H, Lee, B: See-through three-dimensional display using printed holographic-optical-element. In: Digital Holography \& 3-D Imaging Meeting, pp. 2-4. Optical Society of America, (2015)

5. Sinha, A, Barbastathis, G: Broadband volume holographic imaging. Appl. Opt. 43(27), 5214-5221 (2004)

6. Wissmann, P, Oh, SB, Barbastathis, G: Simulation and optimization of volume holographic imaging systems in zemax ${ }^{\circledast}$. Opt. Express. 16(10), 7516-7524 (2008)

7. Kalkum, F: Fast numerical simulation of diffraction from large volume holograms. J. Opt. Soc. Am. A. 26(11), 2393-2397 (2009)

8. Kogelnik, H: Coupled wave theory for thick hologram gratings. Bell Syst. Tech. J. 48(9), 2909-2947 (1969)

9. Luo, Y, Castro, J, Barton, JK, Kostuk, RK, Barbastathis, G: Simulations and experiments of aperiodic and multiplexed gratings in volume holographic imaging systems. Opt. Express. 18(18), 19273-19285 (2010)

10. Syms, R, Solymar, L: Localized one-dimensional theory for volume holograms. Optical \& Quantum Electronics. 13(5), 415-419 (1981)

11. Jurbergs, D, Bruder, F-K, Deuber, F, Fäcke, T, Hagen, R, Hönel, D, Rölle, T, Weiser, M-S, Volkov, A: New recording materials for the holographic industry. In: Proc. SPIE 7233, Practical Holography XXIII: Materials and Applications, pp. 72330-7233010, (2009)

12. Jurbergs, D, Bruder, F-K, Deuber, F, Fäcke, T, Hagen, R, Hönel, D, Rölle, T, Weiser, M-S, Volkov, A: Reaction-diffusion model applied to high resolution bayfol ${ }^{\oplus} \mathrm{hx}$ photopolymer. In: Proc. SPIE 7619, Practical Holography XXIV: Materials and Applications, pp. 76190-7619015, (2010)

13. Berneth, H, Bruder, FK, Fäcke, T, Hagen, R, Hönel, D, Rölle, T, Weiser, M-S, Jurbergs, D: Holographic recording aspects of high-resolution bayfol ${ }^{\circledR} \mathrm{hx}$ photopolymer. In: Proc. SPIE 7957, Practical Holography XXV: Materials and Applications, pp. 79570-7957015, (2011)

14. Berneth, H, Bruder, F-K, Fäcke, T, Hagen, R, Hönel, D, Rölle, T, Walze, G, Weiser, M-S: Holographic recordings with high beam ratios on improved bayfol ${ }^{\circledR}$ hx photopolymer. In: Proc. SPIE 8776, Holography: Advances and Modern Trends III, vol. 8776, pp. 877603-87760312, (2013)

15. Berneth, H, Bruder, F-K, Fäcke, T, Hagen, R, Hönel, D, Rölle, T, Walze, G, Jurbergs, D: Bayfol hx photopolymer for full-color transmission volume bragg gratings. In: Proc. SPIE 9006, Practical Holography XXVIII: Materials and Applications, vol. 9006, pp. 900602-90060210, (2014)

16. Lindlein, N: Analyse und optimierung diffraktiver optischer systeme. Friedrich-Alexander-Universität Erlangen-Nürnberg (1996). PhD thesis

17. Bragg, WL: The diffraction of short electromagnetic waves by a crystal. In: Proceedings of the Cambridge Philosophical Society, pp. 43-57, (1913)

18. Zemax: OpticStudio 16 SP2 Help Files (2016)

\section{Submit your manuscript to a SpringerOpen ${ }^{\circ}$ journal and benefit from:}

- Convenient online submission

- Rigorous peer review

- Open access: articles freely available online

- High visibility within the field

- Retaining the copyright to your article

Submit your next manuscript at $\gg$ springeropen.com 